\title{
RESIDUOS DE CAMARÓN SIETE BARBAS, COMO ABONO ORGÁNICO EN EL CULTIVO DE TOMATE
}

Xiomara Treminio L. ${ }^{[1]}$ Jordi Bartolomé F. ${ }^{[2]}$

\section{Resumen}

La investigación se desarrolló con la finalidad de evaluar el comportamiento de plántulas de tomate (Lycopersiconesculentum mill) de la variedad peto 98 bajo diferentes dosificaciones de abono orgánico elaborado a base de desechos de camarón siete barbas (Xiphopenaeuskroyeri). El experimento se llevó a cabo en la comunidad de Rocky Point, Municipio de Laguna de Perlas, en donde la naturaleza del experimento se cataloga como un arreglo unifactorial, empleándose un diseño experimental de bloques completamente al azar (BCA), con 4 tratamientos y 3 repeticiones. Las variables evaluadas correspondieron a: altura de la planta $(\mathrm{cm})$, diámetro de tallo $(\mathrm{cm})$ y porcentaje de rendimiento $(\mathrm{Kg})$, las variables se sometieron a un análisis de varianza (ANDEVA) con un 95\% de confianza y prueba de rangos múltiples según el criterio de Tukey con 0.05 para la separación de medias. La mayor altura (parte aérea) de las plántulas fue con el tratamiento 3 ( $\mathrm{T}_{3}$ ) con $250 \mathrm{~g} / \mathrm{planta}$, el segundo mejor valor de altura correspondió al tratamiento 2 ( $\mathrm{T}_{2}$ ) con 35og/planta, seguido de los tratamientos 4 ( $\mathrm{T}_{4}$ ), con $150 \mathrm{~g} /$ planta) y tratamiento 1 ( $\mathrm{T}_{1}$ ) como testigo. Con respecto al diámetro se observó que en los primeros 45 días después de la siembra los T3 (250g) y T3 (350), no muestran diferencias significativas siendo los tratamientos en donde se obtuvo un mejor comportamiento, seguido de los $\mathrm{T}_{4}$ (150 g) y 11 (testigo), en cuanto a rendimiento de las plántulas de tomate, el comportamiento fue similar al diámetro en donde los $\mathrm{T}_{2} \mathrm{y} \mathrm{T}_{3}$, presentan mejores rendimientos en cosecha, seguidos por los $\mathrm{T}_{4} \mathrm{y} \mathrm{T} 1$.

Palabra Clave: Abono; camarón; desechos; sostenible; valor agregado.

\section{Summary}

The research was developed in order to evaluate the tomato seedlings behaviour (Lycopersiconesculentum mill) of the variety breastplate 98, under different dosages of compost made from seabob shrimp waste (Xiphopenaeuskroyeri). The experiment took place in the community of Rocky Point, Municipality of Pearl Lagoon, where the nature of the experiment is classified as a unifactorial arrangement, using an experimental design of randomized complete block (RCB) with 4 treatments and 3 repetitions. The variables evaluated were for: height of the plant $(\mathrm{cm})$, stem diameter $(\mathrm{cm})$ and production percentage $(\mathrm{kg})$. The variables were submitted to an analysis of

[1] MSc. URACCAN, Bilwi. Bo El Muelle de FADCANIC 75 vrs al sur. nelsongalosacasa@gmail.com

[2] MSc. Coordinadora NITLAPAN-UCA. Bilwi, Costado Sur del estadio Municipal de Baseboll. diriangenus@yahoo.com 
variance (ANOVA), obtaining 95\% of reliance and multiple range tests according to Tukey's criterion with 0.05 for mean separation. The greatest height of the seedlings (aerial part) was with treatment $3\left(\mathrm{~T}_{3}\right)$ with $250 \mathrm{~g} / \mathrm{plant}$, the second best value of height corresponded to treatment 2 ( $\left.\mathrm{T}_{2}\right)$ with $350 \mathrm{~g} / \mathrm{plant}$, followed by the treatments $4\left(\mathrm{~T}_{4}\right)$ with $150 \mathrm{~g} /$ plant and treatment $1\left(\mathrm{~T}_{1}\right)$ as a baton. Regarding the diameter, we observed that during the first 45 days after sowing, the $\mathrm{T}_{3}(250 \mathrm{~g})$ and $\mathrm{T}_{3}(350)$ showed no significant difference, being the groups that obtained the best behaviour, followed by $\mathrm{T}_{4}\left(150 \mathrm{~g}\right.$ ) and $\mathrm{T}_{1}$ (baton). As for the tomato seedlings behaviour, it was similar to the diameter where $\mathrm{T}_{2}$ and $\mathrm{T}_{3}$ presented better crop production, followed by $\mathrm{T}_{4}$ and $\mathrm{T}_{1}$.

Keyword: Compost; shrimp; waste; sustainable; aggregate value.

\section{Introducción}

La actividad económica principal del Municipio de Laguna de Perlas es la pesca, siendo la comercialización de camarones una de las más importantes fuentes de recursos económicos para la Región. Según el Plan Nacional de Desarrollo Humano (2012-2016), en los últimos años ha aumentado la Producción camaronera en un 60 $\%$ en el período, 2006 al 2012, lo que ha generado un alto porcentaje de desperdicios representados por la cabeza y el caparacho del camarón que es producido durante el proceso de aprovechamiento. El volumen generado, unido a su lenta capacidad de degradación ha estimulado una intensa actividad investigadora centrada en la determinación de los posibles usos de estos recursos con una doble finalidad, la eliminación de un problema medioambiental y la búsqueda de una explotación económica beneficiosa. Estudios anteriores han demostrado que los desechos de camarón pueden ser utilizados como materia prima para la producción de quitina y proteínas (Hernández y Escorcia, 2009). En el 2004, Parada, determinó que son recursos biodegradables en la tierra y biocompatibles con órganos, tejidos y células de animales y plantas por sus propiedades biológicas y químicas; además de contribuir al problema de la disposición de desechos incrementando el interés por la búsqueda de opciones de reducción y de aprovechamiento. La producción de plántulas con el uso de sustratos bajo ambientes controlados ha sido una alternativa útil para cultivos de alta importancia como el tomate, ya que ha permitido incrementar la productividad, además de obtener un producto de mejor calidad, el cual puede ser obtenido con un uso más racional y reducido de insumos, y como consecuencia, un menor daño ambiental (Bracho, 2005).

El tomate (Lycopersicon esculentum Mill) es una hortaliza de fruto de usos múltiples, más importantes de Nicaragua dentro del subsector agrícola vegetal, se cultiva principalmente para el consumo fresco. (MAGFOR, 2007). La incursión de nuevas técnicas de fertilización orgánica, se hacen con la intención de que los agricultores tengan alternativas en la disminución de aplicaciones de fertilizantes químicos, los cuales durante mucho tiempo han perjudicado la calidad ambiental. 
Por lo anterior esta investigación evaluó el comportamiento de plántulas de tomate variedad Peto 98, mediante la aplicación de diferentes dosis de residuos de camarón siete barbas (Xiphopenaeuskroyeri), para determinar si los componentes químicos son nutrientes asimilables y disponibles para las plantas.

\section{Revisión de literatura}

\section{Camarón Xiphopenaeuskroyeri}

De acuerdo a Welder E. (1998) el camarón (Penaeus Sp), es el nombre genérico de crustáceos decápodos nadadores que habitan en agua dulce y en mayor diversidad en el medio marino, el cuerpo está protegido por un exoesqueleto que debe mudar a medida que crece.

En relación al procesamiento, una vez capturado, generalmente son descabezados y entregados a las plantas de procesamiento, para posteriormente prepararlo para introducirlo a la cadena de comercialización (Madrid A., Madrid J. y Madrid R., 1994).

Basado en lo anterior, los subproductos generados pueden dividirse en sólidos y líquidos. Entre los primeros se encuentran: cefalotórax, cutícula o caparazón, vísceras y fragmentos de carne que no han sido removidos en la operación de pelado, mientras que los desechos líquidos o efluentes, están representados por el agua de blanqueo (Caicedo M., 1982). De acuerdo a Shirai K., (1999), el rendimiento de los subproductos cuando se tiene el camarón en forma de cola con cáscara oscila entre 35 y $45 \%$ sobre el peso total del camarón, estos desechos pueden repercutir negativamente en el medio ambiente y pueden generar un costo adicional para su deposición, reduciendo las utilidades del sistema de producción.

\section{Productividad de camarón en Nicaragua}

En Nicaragua la producción de camarón durante el período comprendido entre el 2005 al 2009 fue de 14,061 TM (Solórzano, A, 2010). Según el Plan Nacional de Desarrollo Humano, (2012-2016), en los últimos años ha aumentado la Producción camaronera en un $60 \%$ en el período del 2006 al 2012, por lo que se ha generado un alto porcentaje de desperdicios representados por la cabeza y el caparacho del camarón producido durante el proceso de aprovechamiento.

\section{Abonos orgánicos}


Altieri (1995), expresa que el abono orgánico es un producto natural resultante de la descomposición de materia de origen vegetal, animal y mixto, que tienen la capacidad de mejorar la fertilidad y estructura del suelo, la capacidad de retención de la humedad, activar su capacidad biológica, y por ende mejorar la producción y productividad de los cultivos. Por tal razón la importancia fundamental de su necesidad en el suelo obedece a que los abonos orgánicos son fuente de vida bacteriana sin la cual no se puede dar nutrición de las plantas, además de ser totalmente sano y natural ya que cuentan con millones de microorganismos que transforman a los minerales en elementos comestibles para las plantas.

La aplicación de abonos a base de fermentos naturales está orientada a proporcionar un medio ambiente limpio y balanceado, potenciar la capacidad productiva y fertilidad natural de los suelos, optimizar el reciclaje de los nutrientes, el control natural de plagas y enfermedades.

\section{Tomate (LycopersiconesculentumMill)}

Es una hortaliza de fruto de usos múltiples, más importantes de Nicaragua dentro del subsector agrícola vegetal, (MAGFOR, 2007).La exigencia del tomate en nutrientes especialmente NPK, obliga a buscar técnicas más eficientes de proporcionarlos (Morales, 1999).

\section{Materiales y métodos}

El experimento se realizó en la comunidad de Rocky Point, localizada dentro de las coordenadas geográficas $12^{\circ} 20^{\prime}$ Latitud Norte y $83^{\circ} 40^{\prime}$ Latitud Oeste, ubicada en el Municipio de Laguna de Perlas, RAAS, Nicaragua.

\section{Materia prima}

El abono orgánico se obtuvo del desperdicio de camarón seco molido a partir de desechos de cabezas y caparazón de camarón de estuario (Xiphopenaeus kroyeri), al que se le realizó un análisis de Macronutrientes, mas calcio y magnesio para determinar los componentes nutricionales que contiene.

\section{Procedimiento}

El experimento realizado según la naturaleza se clasifica como un arreglo unifactorial el cual se empleó en un diseño experimental de Bloques Completamente al Azar (BCA), con 4 tratamientos correspondientes a diferentes dosis de abono orgánico (o, 350,250 y $150 \mathrm{~g} /$ planta), estas dosis con secuencia logarítmica fueron escogidas debido a que no existen estudios anteriores con recomendaciones al respecto. 
Cada tratamiento tuvo tres repeticiones, para un total de 12 unidades experimentales (UE). Una UE estuvo compuesta por 10 plantas de tomate y 3 repeticiones que se realizaron al momento de la siembra y las otras dos repeticiones a los 30 y 60 días después de la siembra.

Las evaluaciones se realizaron a los 15, 45 y 75 días después de la siembra a las siguientes variables: Altura de la planta $(\mathrm{cm})$, Diámetro de tallo $(\mathrm{cm})$ y Rendimiento: peso en Kilogramos del cultivo.

Cuadro No. 1: Tratamientos experimentales, incluyendo el control (T1), aplicado al cultivo de tomate

\begin{tabular}{|l|l|l|l|}
\hline \multicolumn{1}{|c|}{ Tratamientos } & \multicolumn{1}{|c|}{ Descripción } & Repeticiones & $\begin{array}{c}\text { Aplicaciones por } \\
\text { Tratamiento }\end{array}$ \\
\hline$T_{1}$ (Testigo) & Cultivo de tomate + control de maleza. & & 3 \\
\hline$T_{2}$ & $\begin{array}{l}\text { Cultivo de tomate }+ \text { control de malezas }+ \\
350 \mathrm{~g} / \text { planta. }\end{array}$ & $3 w x$ & 3 \\
\hline$T_{3}$ & $\begin{array}{l}\text { Cultivo de tomate }+ \text { control de malezas }+ \\
250 \mathrm{~g} / \text { planta }\end{array}$ & 3 & 3 \\
\hline$T_{4}$ & $\begin{array}{l}\text { Cultivo de tomate }+ \text { control de malezas }+ \\
150 \mathrm{~g} / \text { planta }\end{array}$ & 3 & \\
\hline
\end{tabular}

\section{Análisis de datos}

El análisis se realizó con el programa estadístico InfoStat/L; Primeramente las variables se sometieron a un análisis de varianza (ANDEVA) con un 95\% de confianza y prueba de rangos múltiples y en aquellas en donde se encontró diferencias significativas se realizó una prueba de comparación de medias según análisis paramétricas post hot de Tukey $(a=0.05)$.

\section{Resultados y discusión}

Composición química de los residuos de camarón siete barbas (Xiphopenaeuskroyeri).

Los residuos de camarón (Xiphopenaeuskroyeri), al realizarse análisis químico de macro y micro nutrientes se determinó que contiene nutrientes asimilables y disponibles para las plantas de tomate según la exigencia nutricional del cultivo, por lo que estos desechos pueden ser aplicado como abono orgánico, contribuyendo de esta manera a un problema medioambiental y una alternativa económicamente beneficiosa en el Municipio de Laguna de Perlas mediante la utilización de este desecho que ha incrementado en volumen en los últimos años. 
Cuadro No. 2: Composición de nutrientes contenidos en el abono orgánico a base de residuos de camarón (Xiphopenaeuskroyeri)

\begin{tabular}{|l|l|}
\hline \multicolumn{1}{|c|}{ Parámetros físico - químicos } & \multicolumn{1}{c|}{ Resultados } \\
\hline $\mathrm{pH}$ & 8.2 \\
\hline $\mathrm{CE}(\mu \mathrm{S} / \mathrm{cm})$ & 10.62 \\
\hline $\mathrm{MO} \%$ & 66.7 \\
\hline $\mathrm{Nt} \%$ & 3.5 \\
\hline $\mathrm{P} 2 \mathrm{O} 5 \%$ & 1.6 \\
\hline $\mathrm{K} 2 \mathrm{O} \%$ & 8.9 \\
\hline $\mathrm{CaO} \%$ & 20.8 \\
\hline $\mathrm{MgO} \%$ & 0.8 \\
\hline Fuente: LABSUELO/UNAN - León (2013). &
\end{tabular}

Evaluación de los parámetros morfométricos de las plantas, altura y diámetro basal y rendimiento con la aplicación de abono orgánico

\section{Altura}

La altura de la planta es uno de los factores de crecimiento que influye sobre la capacidad fotosintética del cultivo de tomate y hace posible un desarrollo apropiado que determinara la productividad de la planta (Alemán, M. 1991).

En la figura No. 1, se observa el comportamiento de las diferentes variables evaluadas entre los valores medios de la altura de las plantas de tomate a los 15, 45 y 75 días para cada uno de los tratamientos con la aplicación de diferentes dosis de abono orgánico de desechos de camarón se determinó que existe diferencia significativa en el crecimiento de las plantas a los 15 y 75 días de la siembra dado que el valor de significación a los 15 días es ( $\mathrm{p} \leq 0.0045)$; y a los 75 días es ( $\mathrm{p} \leq 0.0141)$, según el análisis de varianza paramétrica post hot de Tukey, a diferencia del comportamiento de las plantas a los 45 días en el cual no mostro diferencias significativas en ninguno de los tratamientos con un valor de significación de ( $\mathrm{p} \leq \mathbf{0 . 0 1 4 1 )}$. Al analizar la variable altura de la planta estuvieron en un rango de 12,94 a 66,94 cm. La mayor altura de la parte aérea de la plántula fue con el T3 (250 gr), los segundos mejores valores de altura se observó en los T2 (350 g) y T4 (150 g). El menor valor de altura se presentó en el $\mathrm{T} 1$ (testigo).

Padilla y Peralta (1994), indica que la altura de la planta se ve afectada por el factor nitrógeno esto coincide con los datos encontrados en este estudio donde las plantas con $\mathrm{T}_{2}(250 \mathrm{~g}), \mathrm{T}_{3}(350 \mathrm{~g})$ y $\mathrm{T}_{4}(150 \mathrm{~g})$, obtuvieron un mayor aumento en altura en comparación con el T1 (testigo), además indican que el tomate desde la siembra hasta la floración extrae del suelo un aproximado del $2 \%$ de NPK, según análisis de macronutrientes realizado a los desechos de camarón se determinó que el Nitrógeno Total (Nt) contenido en el abono es del $3.5 \%$, esto nos indica que el abono contiene 
suficiente nitrógeno para el desarrollo óptimo de las plantas también contiene $1.6 \%$ de Pentoxido de fósforo ( $\mathrm{P}_{2} \mathrm{O}_{5}$ ) y un 8.9\% de Oxido de potasio ( $\left.\mathrm{K}_{2} \mathrm{O}\right)$, y las hortalizas requieren del $6 \%$ para lograr desarrollarse, posee un contenido de materia orgánica o Molibdeno (MO) de 66.7\%, esto ayuda a la fijación simbiótica del nitrógeno(aminoácidos y proteínas) y a reducir los contenidos de nitratos en el cultivo de tomate por lo que se considera un abono rico en humos esta materia orgánica mejora las condiciones físicas, químicas y biológicas del suelo; suaviza y permite una aeración adecuada de las plantas y aumenta la porosidad e infiltración del agua en el suelo, cabe mencionar que según INETER, 2009, los suelos del Municipio son pobres en nutrientes, se caracterizan por ser hondos desde franco arenosos, hasta franco - arcillosos - arenosos altamente meteorizados y desmenuzables, bien drenados, con baja cantidad de materia orgánica y poco material nutritivo para las plantas el $\mathrm{PH}$ oscila entre 5.5 a 6 el cual es muy bajo para el cultivo de tomate, pero con la incorporación de este abono orgánico considerado ligeramente básico con $\mathrm{pH}$ de 8.2, permite la regulación del $\mathrm{PH}$ en el suelo, logrando un crecimiento óptimo de las plantas de tomate.

Estos datos nos indican que independientemente de las dosis aplicadas, la planta extrajo la cantidad de elementos necesarios para alcanzar la altura adecuada y definida genéticamente.
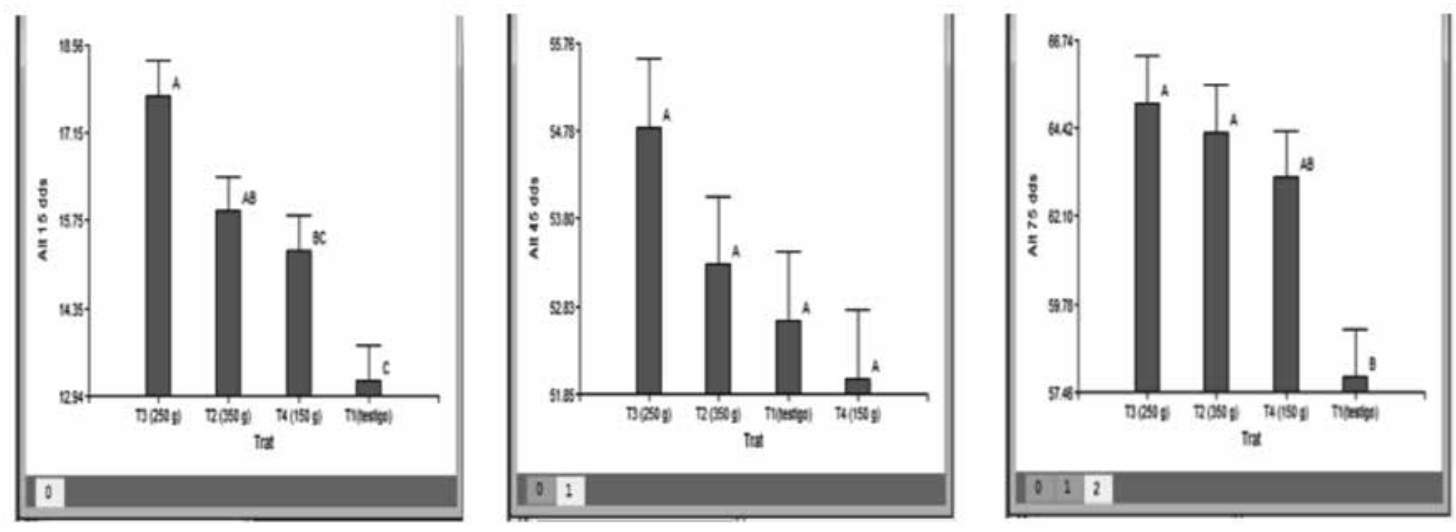

Figura No.1. Diferencias entre los valores medios de la altura a los 15, 45 y 75 días para cada uno de los tratamientos.

\section{Diámetro}

El diámetro del tallo es un elemento dimensional horizontal que resulta del crecimiento y desarrollo de la planta como consecuencia de la formación de nuevas células y de la producción de asimilados (Wild, A. 1992), En la figura No. 2, se reflejan las diferencias significativas en el diámetro del tallo a los 15,45 y 75 días después de la siembra de las plantas de tomate dado que el valor de significación a los 15 días es ( $\mathrm{p} \leq 0.0013$ ), a los 45 dias fue de $(\mathrm{p} \leq 0.0014)$, y a los 75 días ( $\mathrm{p} \leq 0.0013)$, según el análisis de varianza 
paramétrica post hot de Tukey, lo que indica que las dosis aplicadas $\mathrm{T}_{3}(250 \mathrm{~g})$ y $\mathrm{T}_{4}$ (350 g), presentan diámetros mayores de igual medida en los primeros 45 dias, mostrando diferencias significativas con $\operatorname{los}_{4}\left(150 \mathrm{~g}\right.$ ) y $\mathrm{T}_{1}$ (testigo) quienes muestran un mismo comportamiento a los 75 días el $\mathrm{T}_{3}(250 \mathrm{~g})$ obtuvo un mejor comportamiento con diámetro de $2,81 \mathrm{~cm}$, presentando diferencias significativas en el grosor del tallo comparado con $\mathrm{T}_{2}\left(35 \mathrm{~g}\right.$ ), $\mathrm{T}_{4}$ ( $150 \mathrm{~g}$ ) y $\mathrm{T}_{1}$ ( testigo), quienes muestran un porcentaje menor en diámetro.

Esto incide a que el óxido de calcio(Cao) contenido en el abono aplicado corresponde al $20.8 \%$, las plantas de tomate necesitan el $6.5 \%$ para desarrollas sus tallo por lo que los nutrientes contenidos son suficientes para el buen desarrollo del tallo , cabe mencionar que la conductividad eléctrica $C E(\mu S / \mathrm{cm})$ obtenida es de 10.62 microsiemens por centímetro, esto refleja todas las sales disueltas contenidas en el agua, la cual presenta un riesgo bajo ya que es un producto poco salino y no causa ningún problema en la aplicación del cultivo de tomate, los niveles de sales óptimos para dicho cultivo son menores de $250 \mu \mathrm{S} / \mathrm{cm}$.
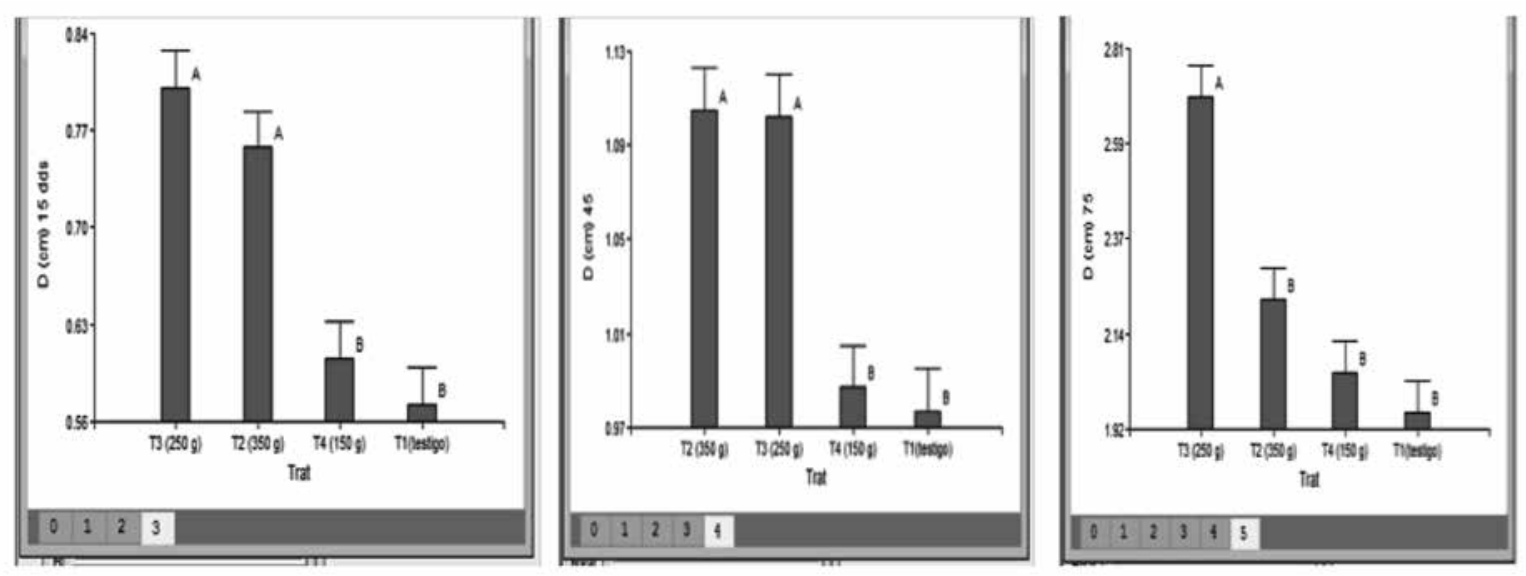

Figura No. 2. Diferencias entre los valores medios del diámetro de la planta a los 15, 45 y 75 días después de la siembra para cada uno de los tratamientos.

\section{Rendimiento}

El fruto del tomate es una baya de forma y tamaño variable dependiendo del número de lóculos que van del 1 al 10 (Avendaño, 1984).La nutrición vegetal es un factor determinante en la producción.

En la figura No. 3, se presenta el comportamiento de los diferentes tratamientos en cuanto al rendimiento en $\mathrm{Kg}$ de las plantas de tomate a los 75 días de siembra con la aplicación de diferentes dosis de abono orgánico de desechos de camarón se determinó que existe diferencia significativa en el rendimiento de las plantas en donde los $\mathrm{T}_{2}$ 
(350 g) y $\mathrm{T}_{3}(250 \mathrm{~g})$, presentan mejores rendimientos en cosecha, seguidos por los $\mathrm{T}_{4}(150 \mathrm{~g}$ ) y T1(testigo), según el análisis de varianza paramétrica post hot de Tukey.

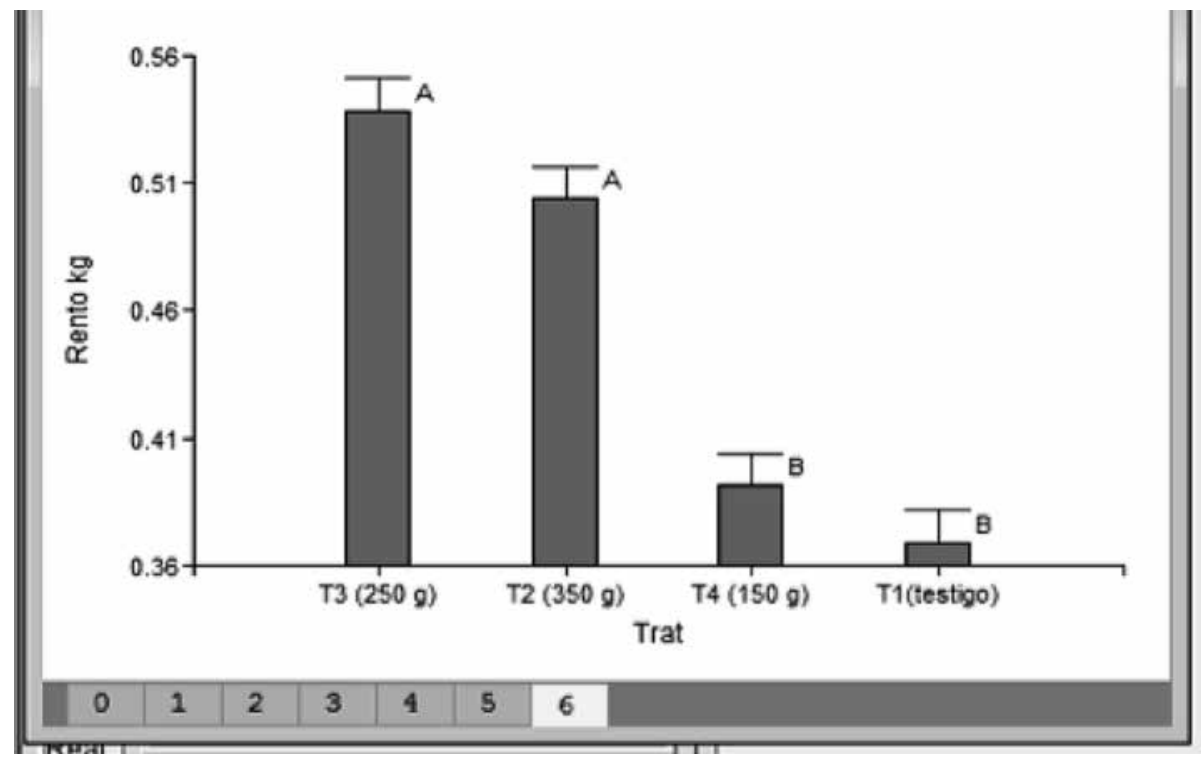

Figura No. 3. Diferencias entre los valores medios de rendimiento en Kg de las planta a los 15, 45 y 75 días después de la siembra para cada uno de los tratamientos.

\section{Conclusiones}

El abono orgánico elaborado a base de residuos de camarón siete barbas (XiphopenaeusKroyeri) contiene nutrientes esenciales para el crecimiento y desarrollo del cultivo de tomate, se considera mesclar este abono con otro tipo de sustrato para evitar crear resistencia.

Las plántulas de tomate variedad peto 98 mostraron diferentes comportamientos en las variables altura, diámetro y rendimiento con la aplicación de diferentes dosis más el testigo, el $\mathrm{T}_{3}(250 \mathrm{~g})$ generó mejor comportamiento en las tres variables evaluadas. Sin embargo, hubo un segundo mejor comportamiento con el T2 (350 g), superando a los $\mathrm{T}_{4}$ (150 g), y $\mathrm{T}_{1}$ (testigo).

Los resultados del estudio nos indican que existe un alto potencial de usos alternativo de este desecho adquiriendo mayor relevancia en la incorporación de procesos de gestión ambiental en el Municipio de Laguna de perlas. 


\section{Lista de referencias}

Alemán, M. (1991). Comportamiento agronómico e industrial de cinco variedades de tomate en el valle de sebaco. Tesis de Ing. ISCA, Managua, Nicaragua.

Altieri, M. (1995). Agroecología: creando sinergia para la agricultura sostenible, Universidad de Berkeley y Consorcio Latinoamérica Agroecología y desarrollo (CLADES). Aplicaciones. Lima, Perú: Fondo Editorial de la Pontifica Universidad Católica del Perú.

Avendaño, S. (1984). El cultivo de tomate (folleto mimeografiado). Universidad Nacional Autónoma de Nicaragua, Managua, Nicaragua.

Bracho, J. (2005). Caracterización de sustratos para la producción de plántulas de tomate (Lycopersiconesculentummill.) En bandejas. Trabajo presentado para optar al grado de Magister Scientiarum. Universidad Centroccidental Lisandro Alvarado. Pp: 91

Caicedo, M. (1982). Aprovechamiento de los desechos del camarón en la elaboración de concentrados proteicos y derivados quitinosos. Santa Marta: Universidad del Magdalena, Colombia.

Hernández, D. \& Escorcia, D. (2009). Propuesta Técnica para la obtención de quitina a partir de caparazones de crustáceos a nivel de plantapiloto. Tesis de Ingeniero Químico. Universidad Nacional de Ingeniería (UNI), Managua, Nicaragua.

INETER, (2009).CaracterizaciónClimática de la Región Autónoma del Atlántico Sur (RAAS).

MADRID, A, MADRID, J. y MADRID, R. (1994). Tecnología del pescado y productos derivados. Iragra S.A. Madrid Vicente ediciones, España.

MAGFOR (2007). Área cosechada, rendimientos y producción de hortalizas a nivel nacional. Ministerio Agropecuario y Forestal. Managua, Nicaragua.

Morales, F, (1999). Cultivo del tomate. Guía tecnológica 22, INTA, Ed.IMPASA.

Padilla y Peralta, I. (1994).Influencia de la fertilidad nitrogenada y densidad de siembra en el rendimiento Agronómico e industrial del tomate. Tesis de Ing. Agraria. UNA, Managua, Nicaragua.

Parada. (2004). Caracterización de quitosano por viscosimetría capilar y valoración Potenciométrica. Revista Iberoamericana de Polimeros, volumen 5. 
Pastor de Abram, A. (2004). Quitina y Quitosano: obtención, caracterización.

Plan Nacional de Desarrollo Humano (2012-2016). República de Nicaragua. Recuperado el 15 de octubre del 2012 de: http://www.pndh.gob.ni/

Richmond, F. (2010). Evaluación de distintas materias primas para la producción de Almácigo de tomate. Agronomía Costarricense 34(1): 85-91.

Shirai, K. (1999). Utilización de desechos de camarón para obtención de quitina, proteínas y pigmentos por vía microbiana [TesisDoctoral]. México: Departamento de Biotecnología, Universidad Autónoma Metropolitana.

Solórzano, A. (2010). Potencialidades de producción agroindustrial. Ministerio de Fomento, Industria y Comercio MIFIC.

Welder, E. (1998). Introducción en la Acuacultura con Énfasis en los Neotrópicos. Litoflash, Santa Marta, Colombia.

Wild, A. (1992). Condiciones del suelo y desarrollo de la planta según Rusell. Ed.Mundiprensa. Madrid, España. 